\title{
Multiple Imputation Technique
}

National Cancer Institute

\section{Source}

National Cancer Institute. Multiple Imputation Technique. NCI Thesaurus. Code C81202.

The substitution of missing data with the average derived from multiple datasets of data that is not missing. 